\title{
Stackelberg and Cournot competition under equilibrium limit pricing
}

Citation for published version (APA):

Haan, M., \& Maks, J. A. H. (1996). Stackelberg and Cournot competition under equilibrium limit pricing. METEOR, Maastricht University School of Business and Economics. METEOR Research Memorandum No. 003 https://doi.org/10.26481/umamet.1996003

Document status and date:

Published: 01/01/1996

DOI:

10.26481/umamet.1996003

Document Version:

Publisher's PDF, also known as Version of record

\section{Please check the document version of this publication:}

- A submitted manuscript is the version of the article upon submission and before peer-review. There can be important differences between the submitted version and the official published version of record.

People interested in the research are advised to contact the author for the final version of the publication, or visit the DOI to the publisher's website.

- The final author version and the galley proof are versions of the publication after peer review.

- The final published version features the final layout of the paper including the volume, issue and page numbers.

Link to publication

\footnotetext{
General rights rights.

- You may freely distribute the URL identifying the publication in the public portal. please follow below link for the End User Agreement:

www.umlib.nl/taverne-license

Take down policy

If you believe that this document breaches copyright please contact us at:

repository@maastrichtuniversity.nl

providing details and we will investigate your claim.
}

Copyright and moral rights for the publications made accessible in the public portal are retained by the authors and/or other copyright owners and it is a condition of accessing publications that users recognise and abide by the legal requirements associated with these

- Users may download and print one copy of any publication from the public portal for the purpose of private study or research.

- You may not further distribute the material or use it for any profit-making activity or commercial gain

If the publication is distributed under the terms of Article $25 \mathrm{fa}$ of the Dutch Copyright Act, indicated by the "Taverne" license above, 


\title{
Stackelberg and Cournot competition under equilibrium limit pricing
}

\author{
Marco Haan \\ Hans Maks \\ University of Limburg
}

\begin{abstract}
In this paper we show that the claim that the price in a Stackelberg model is lower than the price in a Cournot model, does not necessarily hold in an entry-deterrence framework. Using a signaling model of entry deterrence, we show that when post-entry competition is Stackelberg instead of Cournot, this might influence the entry decision of a potential entrant in such a way that expected average price can actually be higher under Stackelberg competition. In a simple framework with linear demand and constant marginal costs, we derive the condition under which this holds.
\end{abstract}




\section{Introduction}

In a simple duopoly model, the price in a Stackelberg equilibrium is lower than that in a Cournot equilibrium ${ }^{1}$. When both firms have constant marginal costs, Stackelberg competition is thus superior from a welfare point of view. In this paper however, we show that Stackelberg competition is not necessarily welfare enhancing in an entry-deterrence framework.

Suppose we have one incumbent firm, which tries to deter entry from one potential entrant. If the original incumbent acts as a Stackelberg leader when entry has taken place, post-entry profits for the entrant will be lower than in case of Cournot competition. Therefore, entry is less attractive. In this paper we show that in a Milgrom \& Roberts (1982) limit pricing framework, average prices might be higher with post-entry Stackelberg competition than they are when post-entry competition is Cournot. The threat that a firm will act as a Stackelberg leader thus decreases welfare relative to Cournot competition, instead of increasing it, as it does in a standard model.

In our model, an incumbent firm tries to deter entry from a potential entrant. The incumbent can have either high or low marginal cost. The potential entrant does not know the incumbent's marginal cost. When it enters, it has to incur some fixed costs which cannot be recouped. We assume that if post-entry competition is Cournot, it is profitable to enter if and only if the incumbent has high cost. The incumbent uses its price in the first period to signal its marginal costs. Milgrom \& Roberts show that in this type of model, limit pricing in the sense of Bain (1949) can occur in equilibrium. We define a limit price as a price set by a monopolist, which is below the static monopoly price. In our model, a low cost incumbent sets a price in the first period to convince the potential entrant that it is not profitable to enter. But the potential entrant can only be convinced of the latter, if the price set is so low that it is just not profitable for a high cost incumbent to set that same price in the first period, instead of just setting its own monopoly price and inducing entry. In this case, a low cost

\footnotetext{
${ }^{1}$ Levin (1988) shows that this is the case when Hahn's (1962) conditions for stability of the Cournot equilibrium hold. Anderson \& Engers (1992) prove it in a hierarchical Stackelberg model with a restricted class of demand functions, which includes linear demand.
} 
incumbent applies limit pricing, by setting a price which is lower than the price which maximizes its first period profit. Under some circumstances however, a high incumbent can mimic a low cost incumbent. In that case, a high cost incumbent applies limit pricing.

When we change the model by assuming that post-entry competition is Stackelberg instead of Cournot, some things change in equilibrium. First, we can have that firms which did consider entry in the Cournot case, do not consider entry in the Stackelberg case. Entry will occur less frequently, enabling the incumbent to set its monopoly price more often. Second, the probability that a high cost incumbent can mimic a low cost one, changes. Third, the limit price the incumbent sets, will change. All these effects influence the market price in the pre- and post-entry period, and thus also the expected average price. In this paper we derive under what circumstances the expected average price will be higher under Stackelberg competition. In those cases the standard result of Stackelberg competition yielding lower prices, no longer holds.

The paper is organized as follows. In section 2 we restate the basic results of a standard duopoly model, with both Cournot and Stackelberg competition. In section 3 we introduce our model. The outcome of the model with Cournot and Stackelberg competition will be derived in section 4 resp. 5. Section 6 compares price and welfare effects in both models, and section 7 concludes the paper.

\section{The standard model}

This section reviews the basic results of both Cournot and Stackelberg competition in a static linear demand model. The setup is the following. We have two firms, $i=1,2$. Marginal costs of firm $i$ are constant and given by $c_{i}$. Market demand is determined by $p=a-b q$, with $q$ quantity, $p$ price, and $a$ and $b$ parameters. With Cournot competition, the firms play a quantity setting game with simultaneous moves. In case of Stackelberg competition, the two firms also play a quantity setting game, but one of the firms, the Stackelberg leader, moves first. We assume that the parameters are such that in both the 
Stackelberg and the Cournot model all firms supply non-negative amounts. In table 1 we list the basic results in both models: the quantity supplied $(q)$ and profit achieved $(\pi)$ by every firm, and the resulting market price $(p)$. In the Stackelberg equilibrium, firm 1 is the leader, and firm 2 the follower.

We will use $\pi_{i}^{C}\left(c_{1}, c_{2}\right)$ to denote the profit of firm $i$ in a static Cournot game when marginal costs of firm 1 are given by $c_{1}$, and those of firm 2 are $c_{j}$. Analogously, $\pi_{1}^{S}\left(c_{1}, c_{2}\right)$ is the profit of a Stackelberg leader when its marginal costs are $c_{1}$, and that of its competitor equal $c_{2}$, and $\pi_{2}^{S}\left(c_{1}, c_{2}\right)$ is the profit of the follower under the same cost configuration. Furthermore, we will use $p^{C}$ for the price in a static Cournot equilibrium, and $p^{S}$ for the price in a static Stackelberg equilibrium.

Levin (1988) shows that the Stackelberg price is always smaller than the Cournot price, provided both firms produce and Hahn's (1962) two conditions for the stability of the Cournot model hold. The latter is clearly the case in our linear setup. From our results in table 1 it is straightforward to show that the Stackelberg price is indeed lower than the Cournot price, since existence of Cournot equilibrium requires $a+c_{2}-2 c_{1}>0$. Using this condition, we can also show that the profit of the Stackelberg leader is higher than its Cournot profit, whereas the profit of the Stackelberg follower is lower than its Cournot profit. For this model we have thus established the following facts:

$$
\begin{aligned}
& p^{S}<p^{C} \\
& \pi_{1}^{S}>\pi_{1}^{C} \\
& \pi_{2}^{S}<\pi_{2}^{C} .
\end{aligned}
$$

\begin{tabular}{|c|c|c|c|c|}
\hline & \multicolumn{2}{|c|}{ COURNOT } & \multicolumn{2}{|c|}{ STACKELBERG } \\
\hline & firm 1 & firm 2 & firm 1 & firm 2 \\
\hline$q$ & $\left(a+c_{2}-2 c_{1}\right) / 3 b$ & $\left(a+c_{1}-2 c_{2}\right) / 3 b$ & $\left(a+c_{2}-2 c_{1}\right) / 2 b$ & $\left(a+2 c_{1}-3 c_{2}\right) / 4 b$ \\
\hline$\pi$ & $\left(a+c_{2}-2 c_{1}\right)^{2} / 9 b$ & $\left(a+c_{1}-2 c_{2}\right)^{2} / 9 b$ & $\left(a+c_{2}-2 c_{1}\right)^{2} / 8 b$ & $\left(a+2 c_{1}-3 c_{2}\right)^{2} / 16 b$ \\
\hline$p$ & $\left(a+c_{1}\right.$ & $\left.+c_{2}\right) / 3$ & $(a+2$ & $\left.+c_{2}\right) / 4$ \\
\hline
\end{tabular}

Table 1: Equilibrium with Cournot and Stackelberg competition 


\section{The Entry-deterrence Model}

In this section we describe our entry deterrence model, which is similar to Milgrom \& Roberts (1982). In the model, an incumbent firm tries to deter entry in a situation where it has more information than the potential entrant. By the decision it makes before entry, the incumbent firm tries to manipulate the potential entrant's assessment of that information. Milgrom and Roberts show that in this context limit pricing in the sense of Bain (1949) can occur. If limit pricing occurs, the incumbent sets a pre-entry price which is lower than its monopoly price in an attempt to convince the potential entrant that entry is not profitable. In this way, Milgrom \& Roberts provide a model in which limit pricing is fully consistent with profit maximization of the incumbent and the potential entrant, both before and after entry might take place ${ }^{2}$.

We consider the following model. There are two periods in which a homogeneous good is supplied. Market demand in each period is given by $p=a-b q$. We have two firms: one incumbent and one potential entrant. Both have constant marginal costs. The potential entrant is able to produce against marginal $\operatorname{costs} \bar{c}$. However, the potential entrant does not know whether the incumbent has the same marginal costs $\bar{c}$, or, because it has more experience in producing the good, the incumbent has succeeded in obtaining the lower marginal cost $\underline{c}$. The probability that the incumbent has the lower marginal cost is given by $\rho$. The incumbent of course knows its true marginal cost. In period 1, the pre-entry period, only the incumbent is producing. It sets a quantity, which we denote by $q^{1}$. Based upon this quantity and its own beliefs, the potential entrant decides whether or not to enter. If it does, competition will take place in period 2 , the post-entry period ${ }^{3}$. If it does not, the incumbent can simply set its monopoly price in the second period. We assume that the potential entrant has to incur fixed cost $F$ upon entry. $F$ is such that, given that post-entry competition is Cournot, it is profitable for the potential entrant to enter if the

\footnotetext{
${ }^{2}$ Why earlier models of limit pricing are not consistent in this sense, is made clear in Friedman (1979). See also Roberts (1987).

${ }^{3}$ The term post-entry thus refers to the period after entry could have taken place. It is not necessary that entry actually has taken place.
} 
incumbent has high marginal cost, but it is not profitable to do so if it has low cost. Both firms maximize the sum of their profits in period 1 and 2. For simplicity we assume that the discount rate is zero. We restrict ourselves to pure strategy equilibria.

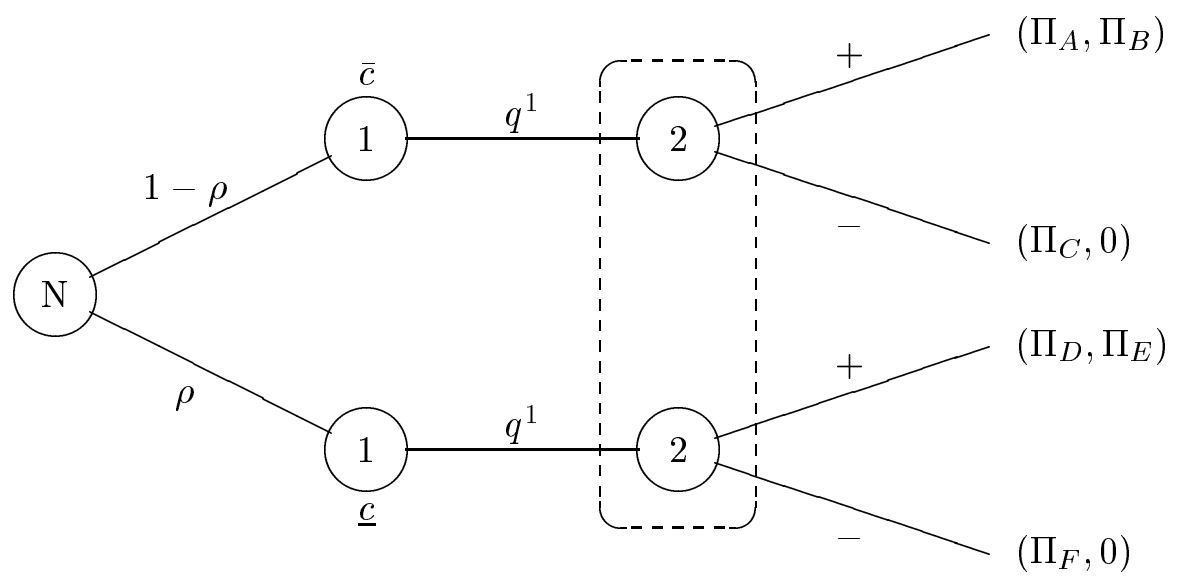

Figure 1: The entry-deterrence game

The game both firms play can be depicted by the game tree in figure 1 . At the first node (on the left hand side) a move by Nature decides whether the incumbent (firm 1) has high or low marginal cost. This choice becomes known to the incumbent firm, which then sets a quantity $q^{1}$ in period 1 . It can choose from a continuum of possible q's, but for simplicity we have represented its decision by a single branch in figure 1 . The potential entrant (firm 2) then observes $q^{1}$, but does not know whether it was set by a high cost or a low cost incumbent, as indicated by the information set. The potential entrant decides to enter $(+)$ or not to enter $(-)$. The resulting payoff vectors in figure 1 will be elaborated upon in the next sections. For each outcome, the first element of the vector represents the payoff to the incumbent firm and the second that of the potential entrant.

To solve this model we look for a sequential equilibrium (see Kreps and Wilson [1982]). Sequential equilibrium requires that the strategy of every player $i$ is rational at each node of the game, given the equilibrium strategies of the other 
players, and given the beliefs the players have at each information set. Moreover, the beliefs must be consistent with the equilibrium strategies. Sequential equilibrium thus requires that the strategies constitute a subgame perfect equilibrium, and that all beliefs are updated according to Bayes' rule.

Our model differs in some respects from Milgrom \& Roberts. They assume that the marginal costs of the potential entrant are unknown to the incumbent firm. We assume they are known, and equal to the high marginal cost of the incumbent firm. This simplifies calculations, and gives an interpretation of the incumbent's marginal cost: we implicitly assume that before entering an industry all firms have access to the same technology, which yields constant marginal costs. However, a incumbent firm which is already producing, might be able to produce more efficiently.

In the next section we will solve for the equilibrium in case post-entry competition is of the Cournot type. In section 5 we do so for post-entry Stackelberg competition.

\section{Equilibrium with Cournot Competition}

We now solve the model outlined in 3 , when post-entry competition is Cournot. We will start by giving the payoffs in figure 1 . To do so, we first introduce some additional notation. First, define $\pi^{1}\left(q^{1}, c_{1}\right)$ as the profit the incumbent firm (firm 1) makes in the first period when it sets a quantity of $q^{1}$. It is easy to see that

$$
\pi^{1}\left(q^{1}, c_{1}\right)=\left(a-b q^{1}-c_{1}\right) q^{1}
$$

Second, as in section 2, we use $\pi_{i}^{C}\left(c_{1}, c_{2}\right)$ to denote firm $i$ 's Cournot profit when marginal costs of firm 1 are $c_{i}$ and that of firm 2 are given by $c_{j}$. Finally, $\pi^{m}\left(c_{i}\right)$ are a firm's monopoly profits when it has marginal cost $c_{i}$, and $q^{m}\left(c_{i}\right)$ is the corresponding monopoly quantity. We can easily show that

$$
\begin{gathered}
q^{m}\left(c_{i}\right)=\left(a-c_{i}\right) / 2 b \\
\pi^{m}\left(c_{i}\right)=\left(a-c_{i}\right)^{2} / 4 b .
\end{gathered}
$$

Consider the upper right-hand branch in figure 1. Here we have that the 
incumbent has high marginal costs, and the potential entrant has decided to enter. In the second period we thus have Cournot competition. Therefore,

$$
\begin{aligned}
& \Pi_{A}=\pi^{1}\left(q^{1}, \bar{c}\right)+\pi_{1}^{C}(\bar{c}, \bar{c}) \\
& \Pi_{B}=\pi_{2}^{C}(\bar{c}, \bar{c})-F .
\end{aligned}
$$

By assumption, we have $\Pi_{B}>0$. In case the incumbent has high cost and the potential entrant does not enter, the latter necessarily has pay-off 0 . The incumbent can set a monopoly price in period 2 , hence we have

$$
\Pi_{C}=\pi^{1}\left(q^{1}, \bar{c}\right)+\pi^{m}(\bar{c})
$$

In the lower half of figure 1, we have similar payoffs, with the difference that the incumbent then has low marginal cost. Along similar lines, we can then show

$$
\begin{aligned}
& \Pi_{D}=\pi^{1}\left(q^{1}, \underline{c}\right)+\pi_{1}^{C}(\underline{c}, \bar{c}) \\
& \Pi_{E}=\pi_{2}^{C}(\underline{c}, \bar{c})-F \\
& \Pi_{F}=\pi^{1}\left(q^{1}, \underline{c}\right)+\pi^{m}(\underline{c})
\end{aligned}
$$

where $\Pi_{E}<0$.

After having defined the payoffs in figure 1, we now solve for the equilibrium in this model. As usual, we do so using backwards induction. At the last node, the potential entrant must decide whether or not to enter. It would want to enter if the incumbent is of the high cost type. However, this is unknown to the potential entrant. It will make its decision based on the belief it has that the incumbent is of the low cost type. We call this belief $\mu ; \mu$ is thus the probability the potential entrant attaches to the event that the incumbent is of the low cost type. Given that belief, the decision to enter is an easy one. Entering will result in a profit of $\Pi_{E}$ with probability $\mu$, and a profit of $\Pi_{B}$ with probability $1-\mu$. Not entering yields zero profits. The potential entrant thus enters iff

$$
\mu \Pi_{E}+(1-\mu) \Pi_{B} \geq 0
$$

The next step is to determine the potential entrant's beliefs. In order to do that we first note that we can have two types of equilibria. In a pooling 
equilibrium, an incumbent always sets the same quantity in the first period, regardless of its type: $q^{1}(\bar{c})=q^{1}(\underline{c})$. In that case, the potential entrant does not obtain any additional information by observing $q^{1}$, since both types of incumbent set the same quantity in period 1. Its belief that it faces a low cost incumbent thus simply equals the a priori probability that an incumbent has low cost: $\mu=\rho$. The other equilibrium is a separating one. In a separating equilibrium the quantity the incumbent sets in the first period does depend on its type: $q^{1}(\bar{c}) \neq q^{1}(\underline{c})$. Upon observing $q^{1}$, the potential entrant thus knows which type of incumbent it faces. Its beliefs are thus $\mu=1$ when it observes $q^{1}(\underline{c})$, and $\mu=0$ when it observes $q^{1}(\bar{c}) .{ }^{4}$

After deriving the beliefs and strategy of the potential entrant in period 2, we now derive the strategy of the incumbent firm in period 1. Consider a low cost incumbent. If the potential entrant had full information, the incumbent would simply set its monopoly quantity $q^{m}(\underline{c})$ in period 1 . The potential entrant would then decide not to enter, for it is not profitable to do so when it faces a low cost incumbent. The incumbent could then also set its monopoly quantity in period 2. However, in this model the potential entrant has incomplete information. If setting $q^{m}(\underline{c})$ would deter entry, then a high cost incumbent might also set $q^{m}(\underline{c})$ and enjoy a monopoly in period $2^{5}$. In this case, the incumbent would fool the potential entrant into thinking that it has low cost, by mimicking the behavior of a low cost incumbent.

Suppose that $\rho$ is such that the potential entrant does not enter in a pooling equilibrium. Since in a pooling equilibrium $\mu=\rho$, we have from (7) that this is the case iff

$$
\rho>\frac{\Pi_{B}}{\Pi_{B}-\Pi_{E}} .
$$

We will refer to the right hand side of 8 as $\rho^{*}$. Suppose (8) does hold. In that

\footnotetext{
${ }^{4}$ The only remaining problem is to specify the potential entrant's beliefs when the incumbent firm takes an out-of-equilibrium action. Suppose the potential entrant observes a $q^{1}$ which neither type of incumbent was allowed to choose in equilibrium. We will follow Cho \& Kreps (1987) in assuming that the incumbent will never send a dominated message, which in this case means that in period 1 an incumbent will never choose a quantity which is always dominated by a different quantity, regardless of the action of the potential entrant in period 2 .

${ }^{5}$ It is more profitable for a high cost incumbent to do this, than it is to set its own monopoly quantity in period 1, and having a Cournot profit in period 2. See appendix.
} 
case a potential entrant will not enter in a pooling equilibrium. Both types of incumbent can then safely set $q^{m}(\underline{c})$ in period 1 . The potential entrant does not enter since it runs too high a risk that the incumbent is of the low cost type. A high cost incumbent sets $q^{m}(\underline{c})$ instead of its own monopoly quantity $q^{m}(\bar{c})$, since the latter will induce entry, and we assumed that a high cost incumbent has a higher profit by setting $q^{m}(\underline{c})$ and deterring entry, than it has by setting $q^{m}(\bar{c})$ and inducing it.

Suppose now (8) does not hold. The potential entrant then enters in a pooling equilibrium. The case in which both types of incumbent set $q^{m}(\underline{c})$ in period 1 is now no longer an equilibrium. A low cost incumbent prefers to set a different quantity, which signals that it is a low cost incumbent, and thus deters entry. It will therefore set a quantity for which it is just not profitable for a high cost incumbent to mimic it. We call this quantity $\hat{q}$. If a high cost incumbent mimics a low-cost one by setting $\hat{q}$ in period 1 , it will deter entry. If it sets its monopoly quantity $q^{m}(\bar{c})$, it does not. From figure 1 , we can see that $\hat{q}$ should satisfy

$$
\Pi_{A}\left(q^{m}(\bar{c})\right)=\Pi_{C}(\hat{q}),
$$

where the argument of both functions denotes the quantity $q^{1}$ set in period 1 . Using (4) and (5), we have that (9) holds iff

$$
\pi^{m}(\bar{c})+\pi_{1}^{C}(\bar{c}, \bar{c})=\pi^{1}(\hat{q}, \bar{c})+\pi^{m}(\bar{c}) .
$$

Using (2) and table 1 we can show that this implies

$$
\hat{q}=\left(1+\frac{1}{3} \sqrt{5}\right)(a-\bar{c}) / 2 b .
$$

Only when a low cost incumbent sets this $\hat{q}$, it can convince the potential entrant that it is of the low cost type, since it is not profitable for a high cost incumbent to mimic this strategy. The latter is better off setting its monopoly quantity in the pre-entry period. We thus have that for any $q^{1} \geq \hat{q}$, the potential entrant will be convinced that the incumbent is of the low cost type ${ }^{6}$. The best a high cost incumbent can do is thus simply set its monopoly quantity in period 1, which induces entry and yields Cournot profits in the pre-entry period.

\footnotetext{
${ }^{6}$ Applying Cho and Kreps' Intuitive Criterion
} 
Thus, when the potential entrant enters in a pooling equilibrium, a low cost incumbent can only deter entry when it sets $\hat{q}$. Note that $\hat{q}$ is larger than a low cost incumbent's monopoly quantity ${ }^{7}$. This implies that a low cost incumbent sets a lower price than its monopoly price. A low cost incumbent thus applies limit pricing. If the potential entrant does not enter in a pooling equilibrium, a high cost incumbent would apply limit pricing: it sets the quantity $q^{m}(\underline{c})$, which is larger than its monopoly quantity $q^{m}(\bar{c})$.

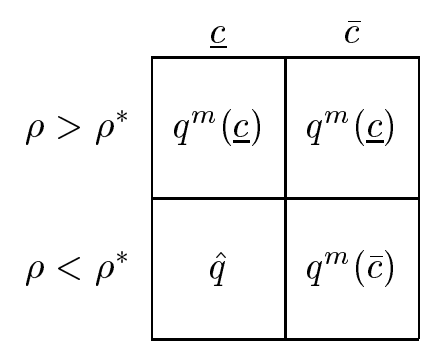

Table 2: $q^{1}$ in equilibrium.

We can summarize the results in this section by table 2. The two columns give the possible type of the incumbent, the rows denote whether or not $\rho<\rho^{*}$ holds. The entries in the table give the quantity each type of incumbent sets in the first period. Here we again see that with $\rho>\rho^{*}$, we have a pooling equilibrium. Both types of incumbent then set quantity $q^{m}(\underline{c})$. The potential entrant cannot observe whether it faces a low cost or a high cost incumbent. Since the risk is too high that the incumbent is of the low cost type, the potential entrant decides not to enter. In this case, by mimicking the behaviour of a low cost incumbent, a high cost incumbent can deter entry. When $\rho<\rho^{*}$ however, the strategies mentioned in the top row of table 1 can no longer constitute an equilibrium. When both types of incumbent would set $q^{m}(\underline{c})$, the potential entrant would enter. Therefore, a low cost incumbent has an incentive to set that quantity which distinguishes it from a high cost incumbent, that is, the quantity $\hat{q}$, where a high cost incumbent is better of setting its own monopoly quantity and inducing entry, than it is setting $\hat{q}$ and deterring it. The best a high cost incumbent can do is then simply setting $q^{m}(\bar{c})$. In equilibrium the

\footnotetext{
${ }^{7}$ Proof in appendix.
} 
potential entrant will enter either if $\rho>\rho^{*}$ and $q^{1}<q^{m}(\underline{c})$, or if $\rho>\rho^{*}$ and $q^{1}<\hat{q}$.

\section{Equilibrium with Stackelberg Competition}

In the previous section we derived an equilibrium for the case in which postentry competition is Cournot. First, we saw that a potential entrant only considers entry when its fixed cost of entry $F$ are smaller than the maximum profit entry can result in ${ }^{8}$. Second, when both types of incumbent set the same quantity in the first period, the entry decision will depend on the probability that the incumbent is of the low cost type. Third, if a potential entrant would decide to enter in such a pooling equilibrium, a low cost incumbent sets a limit price which cannot be profitably set by a high cost incumbent.

In this section we show in which ways the equilibrium changes when postentry competition is Stackelberg instead of Cournot. We will show first that, with post-entry Stackelberg competition, there is a lower probability that a potential entrant considers entry. Second, even if the potential entrant does consider entry, the probability that it will enter in a pooling equilibrium is lower. Third, the limit price set by a low cost incumbent will be higher.

We start the analysis with redefining the variables used in figure 1 . This is a straightforward change in the analysis in section 4 . We now have

$$
\begin{aligned}
& \Pi_{A}=\pi^{1}\left(q^{1}, \bar{c}\right)+\pi_{1}^{S}(\bar{c}, \bar{c}) \\
& \Pi_{B}=\pi_{2}^{S}(\bar{c}, \bar{c})-F \\
& \Pi_{C}=\pi^{1}\left(q^{1}, \bar{c}\right)+\pi^{m}(\bar{c}) \\
& \Pi_{D}=\pi^{1}\left(q^{1}, \underline{c}\right)+\pi_{1}^{S}(\underline{c}, \bar{c}) \\
& \Pi_{E}=\pi_{2}^{S}(\underline{c}, \bar{c})-F \\
& \Pi_{F}=\pi^{1}\left(q^{1}, \underline{c}\right)+\pi^{m}(\bar{c}) .
\end{aligned}
$$

When we repeat the analysis of the previous section, some things are changed. First, we now have that the upper bound on fixed costs $F$ to make entry attrac-

\footnotetext{
${ }^{8}$ Later in this paper we will also say that the potential entrant considers entry when its fixed cost of entry $F$ are smaller than the maximum profit entry can result in.
} 
tive in the first place, will be lower. Note that in section 3 we have assumed that $F$ is such that it is profitable for the potential entrant to enter if the incumbent has high marginal costs, and post-entry competition is Cournot. In other words, we assumed that $F<\pi_{2}^{C}(\bar{c}, \bar{c})$. But if the incumbent has high cost, gross postentry profit with Stackelberg competition, equals $\pi_{2}^{S}(\bar{c}, \bar{c})$. This, from (1), is lower than $\pi_{2}^{C}(\bar{c}, \bar{c})$. Therefore, if $F$ satisfies $\pi_{2}^{S}(\bar{c}, \bar{c})<F<\pi_{2}^{C}(\bar{c}, \bar{c})$, the potential entrant would never consider entry with Stackelberg competition, whereas it would with Cournot competition. If this is the case, the potential entrant can thus always set its monopoly quantity in both the pre- and post-entry period.

Second, if post-entry competition is Stackelberg, $\rho^{*}$ also changes. In (8) $\rho^{*}$ was defined as that $\rho$ for which the potential entrant is just indifferent between entering and not entering in a pooling equilibrium: $\rho^{*}$ equals $\frac{\Pi_{B}}{\Pi_{B}-\Pi_{E}}$. From (4), (12) and (1) we have that both $\Pi_{B}$ and $\Pi_{E}$ are smaller under Stackelberg competition than under Cournot competition. This implies that with Stackelberg competition $\rho^{*}$ is smaller ${ }^{9}$. In other words, there is a larger range of $\rho$ 's for which the potential entrant will not enter in a pooling equilibrium. Therefore, a high cost incumbent will now apply its limit price $q^{m}(\underline{c})$ more often, whereas a low cost incumbent will apply its limit price $\hat{q}$ less often.

Third, suppose that a low cost incumbent does set its limit price. From (9) and (12) we now have that

$$
\pi_{1}^{S}(\bar{c}, \bar{c})=\pi^{1}(\hat{q}, \bar{c})
$$

Since $\pi_{1}^{S}>\pi^{C}$, and $\pi^{1}$ is decreasing in $\hat{q},{ }^{10}$ we have that under Stackelberg competition $\hat{q}$ is smaller than under Cournot competition. If a low cost incumbent sets a limit price, this price will thus be higher under Stackelberg competition. In that case $\hat{q}$ equals

$$
\hat{q}=\left(1+\frac{1}{2} \sqrt{2}\right)(a-\bar{c}) / 2 b .
$$

Summing up, we have that under Stackelberg competition the potential entrant will be less inclined to consider entry. If it does consider entry, it will be less inclined to enter in a pooling equilibrium. If a low cost incumbent sets

\footnotetext{
${ }^{9}$ Proof in appendix

${ }^{10}$ Since we have $\hat{q}>q^{m}(\underline{c})$, and $\pi^{1}$ strictly concave.
} 
a limit price, this limit price will be higher. Note that in the case a potential entrant still considers entry, we can again use table 2 to describe the equilibrium. The only difference is that under Stackelberg competition both $\rho^{*}$ and $\hat{q}$ are lower.

\section{Cournot and Stackelberg compared}

In this section we consider the ultimate effect on price and welfare of both Stackelberg and Cournot competition. To do this, we use the results derived in the previous sections. Since marginal costs are constant, a decrease in price unambiguously increases welfare, either defined as consumer surplus, or as the sum of consumer surplus and firm profits. When we again use a discount rate of zero, the average price in the pre- and post-entry period is thus an unambiguous measure of discounted welfare. As our ultimate measure of welfare we therefore use the expected average price, taking into account that the incumbent will have low marginal costs with probability $\rho$, and high marginal costs with probability $1-\rho$.

We will use the situation with post-entry Cournot competition as a starting point and consider what happens if post-entry competition becomes Stackelberg instead. For simplicity we will refer to the model with post-entry Cournot competition as the Cournot model and to the model with post-entry Stackelberg competition as the Stackelberg model. Analogously, the Cournot equilibrium is the equilibrium in the Cournot model, and the Stackelberg equilibrium the equilibrium in the Stackelberg model.

First consider $F$. In the Cournot equilibrium we had $\pi_{2}^{C}(\underline{c}, \bar{c})<F<$ $\pi_{2}^{C}(\bar{c}, \bar{c})$. In a Stackelberg equilibrium we need $\pi_{2}^{S}(\underline{c}, \bar{c})<F<\pi_{2}^{S}(\bar{c}, \bar{c})$. For any $F$ with satisfies $\pi_{2}^{S}(\bar{c}, \bar{c})<F<\pi_{2}^{C}(\bar{c}, \bar{c})$, we thus have that a potential entrant would consider entry in a Cournot model, whereas it would not in a Stackelberg model. This unambiguously raises expected average price. When a potential entrant considers entry, either a high cost or a low cost incumbent will set a limit price in the pre-entry period, as we saw in table 2 . In case the potential entrant does not consider entry, the incumbent can simply set its 
monopoly quantity in both the first and the second period. Expected average price will then be higher.

Note that for the potential entrant to consider entry in both a Stackelberg and a Cournot equilibrium, we need

$$
\pi_{2}^{C}(\underline{c}, \bar{c})<F<\pi_{2}^{S}(\bar{c}, \bar{c})
$$

Such an $F$ cannot exist when $\pi_{2}^{C}(\underline{c}, \bar{c})>\pi_{2}^{S}(\operatorname{barc}, \bar{c})$. Using table 1 it is easy to see that this is the case iff

$$
\Delta c<(a-\bar{c}) / 4
$$

with $\Delta c=\bar{c}-\underline{c}$ the difference between high and low marginal costs. Thus, if (16) holds, expected average price will be higher under Stackelberg competition. Now suppose (15) does hold. We then have that a potential entrant would consider entry in both a Cournot and a Stackelberg model. What happens to expected average price, and thus to welfare, now depends on $\rho$. In the previous section we showed that $\rho^{*}$, defined as that $\rho$ for which a potential entrant is just indifferent between entering and not entering in a pooling equilibrium, is lower in the Stackelberg model than it is in the Cournot model. We thus have $\rho^{* S}<\rho^{* C}$, where the extra superscripts again denote either the Stackelberg or the Cournot model. We can thus have three possibilities for $\rho$ : either $\rho<\rho^{* S}$, or $\rho^{* S}<\rho<\rho^{* C}$, or $\rho>\rho^{* C}$.

First suppose $\rho>\rho^{* C}$. We are then in the upper row of table 2 , in both the Stackelberg and the Cournot case. Both types of incumbent then set $q^{m}(\underline{c})$ in the pre-entry period, and deter entry in that way. In this case the same happens in both the Stackelberg and the Cournot model, and the expected average price will be the same.

Next suppose $\rho<\rho^{* S}$. In that case we are, in both the Stackelberg and the Cournot model, in the lower row of table 2. A low cost incumbent now sets the limit quantity $\hat{q}$, which deters entry, whereas a high cost incumbent set its monopoly quantity $q^{m}(\bar{c})$, which induces entry. Suppose the incumbent has low costs. It then sets a higher pre-entry price in a Stackelberg model than in a Cournot model, since the limit price is higher under Stackelberg competition. 
In the post-entry period the two models yield the same result: in both cases the low cost incumbent sets it monopoly price. When the incumbent turns out to be a low cost one, we thus have here that average price is higher in the Stackelberg model. Now suppose the incumbent has high costs. In the first period it sets its monopoly quantity, in both the Cournot and the Stackelberg model. Then entry takes place, and we have a post-entry price which is higher in the Cournot model then it is in the Stackelberg model. When the incumbent turns out to be a high cost one, we thus have here that average price is lower in the Stackelberg model. Since average price is lower when the incumbent turns out to have high cost, and higher when it turns out to have low cost, the effect on the expected average price depends on $\rho$. There is a $\tilde{\rho}$ such that average expected price is lower in the Stackelberg model whenever $\rho<\tilde{\rho}$, and higher whenever $\rho>\tilde{\rho}$, provided of course that $\tilde{\rho}<\rho^{* S}$.

Finally, suppose $\rho^{* S}<\rho<\rho^{* C}$. We then have that in the Stackelberg model we are in the upper row of table 2 , whereas in the Cournot model we are in the lower row. We thus have that a low cost incumbent in the Stackelberg model sets its monopoly quantity in both periods, but a low cost incumbent in the Cournot model sets its limit price in the pre-entry period. The average price with a low cost incumbent is then higher in the Stackelberg model. When the incumbent is of the high cost type, things are more complicated. We then have that if $\Delta c<(a-\bar{c}) / 3$, the average price for a high cost incumbent is also higher in the Stackelberg model. Therefore, in that case, the expected average price is higher as well ${ }^{11}$. However, if this condition does not hold, the Stackelberg price will be lower for a high cost incumbent. In that case we have a $\bar{\rho}$ such that expected average price in the Stackelberg model is higher if $\rho>\bar{\rho}$, provided of course that $\rho$ is in the relevant interval.

We thus have the following theorem:

Theorem 1 The expected average price will be higher in a Stackelberg equilibrium than in a Cournot equilibrium if any one of the following conditions holds:

\footnotetext{
${ }^{11}$ See appendix.
} 
1. $\Delta c<(a-\bar{c}) / 4$,

2. $F>\pi_{2}^{S}(\bar{c}, \bar{c})$,

3. $\rho<\rho^{* S}$ and $\rho>\tilde{\rho}$,

4. $\rho^{* S}<\rho<\rho^{* C}$ and $\Delta c<(a-\bar{c}) / 3$,

5. $\rho^{* S}<\rho<\rho^{* C}$ and $\rho>\bar{\rho}$,

with

$$
\begin{aligned}
\Delta c & =\bar{c}-\underline{c} \\
\pi^{* C} & =\frac{\pi_{2}^{C}(\bar{c}, \bar{c})-F}{\pi_{2}^{C}(\bar{c}, \bar{c})-\pi_{2}^{C}(\underline{c}, \bar{c})} \\
\pi^{* S} & =\frac{\pi_{2}^{S}(\bar{c}, \bar{c})-F}{\pi_{2}^{S}(\bar{c}, \bar{c})-\pi_{2}^{S}(\underline{c}, \bar{c})} \\
\tilde{\rho} & =\frac{1}{1+2 \sqrt{5}-3 \sqrt{2}} \\
\bar{\rho} & =\frac{3 \Delta c-(a-\bar{c})}{(\sqrt{5}-1)(a-\bar{c})}
\end{aligned}
$$

Proof: see appendix.

\section{Conclusion}

In this paper we showed that the claim that the price in a Stackelberg model is lower than the price in a Cournot model, does not necessarily hold in an entry-deterrence framework. Using a signaling model of entry deterrence, we showed that when post-entry competition is Stackelberg instead of Cournot, this might influence the entry decision of a potential entrant in such a way that expected average price can actually be higher under Stackelberg competition. In a simple framework with linear demand and constant marginal costs, we derived the conditions under which this holds.

\section{References}

Anderson, S.P. and M. Engers, 1992, "Stackelberg versus Cournot oligopoly equilibrium", International Journal of Industrial Organisation, pp. 127- 
135,

BAIn, J., 1949, "A Note on Pricing in Monopoly and Oligopoly", American Economic Review, pp. 448-64, Stackelberg Perfect Equilibria", International Economic Review, pp. 61-71,

Cho, I.-C., and D.M. Kreps, 1987, "Signaling Games and Stable Equilibria", Quarterly Journal of Economics, pp. 179-221,

Friedman, J., 1979, "On Entry Preventing Behavior", in Applied Game Theory, ed. by S.J. Brams, A. Schotter and G. Schwodiauer, Vienna, pp. $236-253$,

Hahn, F.H., 1962, "The Stability of the Cournot Oligopoly Solution", Review of Economic Studies, pp. 329-31,

Kreps, D.M., and R. Wilson, 1982, "Sequential Equilibria", Econometrica, pp. $863-894$,

Levin, D., 1988, "Stackelberg, Cournot and collusive monopoly: Performance and Welfare Comparisons", Economic Inquiry, pp. 317-330,

Milgrom P., and J. Roberts, 1982, "Limit Pricing and Entry under Incomplete Information: An Equilibrium Analysis", Econometrica, pp. 443-457, T. Bewley (ed.), Advances in Economic Theory,

\section{Appendix}

In this appendix we prove some of the statements made in the main text. First, we prove that we always have $\hat{q}>q^{m}(\bar{c})$. Second, we prove that it is more profitable for the high cost incumbent to set $q^{m}(\underline{c})$ and deter entry, than to set $q^{m}(\bar{c})$ and induce entry. Third, we prove that a low cost incumbent will always prefer setting $\hat{q}$ and deterring entry, then setting $q^{m}(\underline{c})$ and inducing it. Then we prove that $\rho^{* S}<\rho^{* C}$. Finally, we prove theorem 1.

First, in section 4 we claimed that the following proposition holds.

Proposition 1 In a Cournot equilibrium we always have $\hat{q}>q^{m}(\underline{c})$.

Proof. Suppose $\hat{q}<q^{m}(\underline{c})$. From (11) and (3) we have that this is the case if and only if

$$
\left(1+\frac{1}{3} \sqrt{5}\right) \frac{a-\bar{c}}{2 b}<\frac{a-\underline{c}}{2 b},
$$


which is equivalent with

$$
\Delta c>\frac{1}{3} \sqrt{5}(a-\bar{c}) .
$$

From the assumption that the Stackelberg equilibrium exists however, we have $q_{2}^{S}\left(c_{2}, c_{1}\right)>$ 0 , which implies from table 1 that $a+2 \underline{c}-3 \bar{c}>0$. The latter condition is equivalent with

$$
\Delta c<\frac{1}{2}(a-\bar{c}) .
$$

Since $\frac{1}{3} \sqrt{5}>\frac{1}{2},(20)$ implies that (19) can never hold, which proves the proposition.

Proposition 1 immediately implies

Proposition 2 In the Cournot model it is more profitable for the high cost incumbent to set $q^{m}(\underline{c})$ and deter entry, than it is to set $q^{m}(\bar{c})$ and induce it.

PROOF. $\hat{q}$ is, by definition, that $q^{1}$ for which a high cost incumbent is just indifferent between on the one hand setting that quantity and deterring entry, and on the other hand, setting $q^{m}(\bar{c})$ and inducing entry. The profit function of a high cost incumbent is decreasing for $q^{1}>q^{m}(\bar{c})$. Since $q^{m}(\underline{c})>q^{m}(\bar{c}), \hat{q}>q^{m}(\bar{c})$ implies proposition 2 .

For the equilibrium in section 4 to hold we also need, apart from the conditions mentioned in the text, that a low cost incumbent prefers setting $\hat{q}$ and deterring entry, above setting $q^{m}(\underline{c})$ and inducing entry. We thus need

Proposition 3 In the Cournot model the following condition holds:

$$
\pi^{1}(\hat{q}, \underline{c})+\pi^{m}(\underline{c})>\pi^{m}(\underline{c})+\pi_{1}^{C}(\underline{c}, \bar{c}) .
$$

Proof. Subtracting $\pi^{m}(\underline{c})$ from both sides and using (2) and table 1, we obtain that the condition in the lemma is equivalent with

$$
(a-b \hat{q}-\underline{c}) \hat{q}>(a+\bar{c}-2 \underline{c})^{2} / 9 b,
$$

which, using (11), is equivalent with

$$
\frac{1}{2}\left(1+\frac{1}{3} \sqrt{5}\right)(a-\bar{c}) \Delta c>\frac{4}{9}\{\Delta c+(a-\bar{c})\} .
$$

This holds if and only if

$$
0<\Delta c<\frac{1}{8}(1+3 \sqrt{5})(a-\bar{c})
$$

Since $\frac{1}{2}<\frac{1}{8}(1+3 \sqrt{5})$, condition (20) implies that (24) always holds, which proves the proposition.

Next, we prove

Proposition $4 \rho^{* S}<\rho^{* C}$.

PROOF. From (8) we have in general

$$
\rho^{*}=\frac{\Pi_{B}}{\Pi_{B}-\Pi_{E}} .
$$

Using (4) and (6) for the Cournot case and (12) for the Stackelberg case, we have

$$
\begin{aligned}
\rho^{* C} & =\frac{\pi_{2}^{C}(\bar{c}, \bar{c})-F}{\pi_{2}^{C}(\bar{c}, \bar{c})-\pi_{2}^{C}(\underline{c}, \bar{c})} \\
\rho^{* S} & =\frac{\pi_{2}^{S}(\bar{c}, \bar{c})-F}{\pi_{2}^{S}(\bar{c}, \bar{c})-\pi_{2}^{S}(\underline{c}, \bar{c})} .
\end{aligned}
$$


Using table 1, this simplifies to

$$
\begin{aligned}
\rho^{* C} & =\frac{(a-\bar{c})^{2}-9 b F}{\Delta c\{2(a-\bar{c})-\Delta c\}} \\
\rho^{* S} & =\frac{(a-\bar{c})^{2}-16 b F}{\Delta c\{4(a-\bar{c})-4 \Delta c\}}
\end{aligned}
$$

Note that the numerator of $\rho^{* S}$ is smaller than that of $\rho^{* C}$. Moreover, we have that the denominator of $\rho^{* S}$ minus the denominator of $\rho^{* C}$ equals $\Delta c\{2(a-\bar{c})-3(\Delta c)\}$, which is larger than zero because of condition (20). Therefore, the denominator of $\rho^{* S}$ is larger than that of $\rho^{* C}$. Since the numerator of $\rho^{* S}$ is smaller, and the denominator is larger, we necessarily have $\rho^{* S}<\rho^{* C}$, which proves the proposition.

Finally, we prove the theorem in section 6 . The theorem consists of 5 conditions, which we will prove in that same order.

1. In the text we already proved that $\Delta c<(a-\bar{c}) / 4$ implies $\pi_{2}^{C}(\underline{c}, \bar{c})>\pi_{2}^{S}(\bar{c}, \bar{c})$. This means that there cannot exist an $F$ such that the potential entrant considers entry in both the Cournot and the Stackelberg model. If the potential entrant does not consider entry, we have that the expected average price in the Stackelberg equilibrium will be higher than that in the Cournot equilibrium, which proves that condition 1 is sufficient for the theorem to hold.

2. When $F>\pi_{2}^{S}(\bar{c}, \bar{c})$, the potential entrant does not consider entry in the Stackelberg model, whereas we assumed that it did in the Cournot model. Using the same argument as in condition 1 , we thus have that condition 2 is also sufficient for the theorem to hold.

3. Suppose conditions 1 and 2 do not hold, and $\rho<\rho^{* S}$. In that case we are, both in the Stackelberg and the Cournot model, in the lower row of table 2. This means that a low cost incumbent now sets the limit quantity $\hat{q}$ and deters entry, whereas a high cost incumbent sets $q^{m}(\bar{c})$ and induces entry. We have that price is a linear function of quantity: $p(q)=a-b q$. We denote the average price in case the incumbent has low costs, by $\bar{p}(\underline{c})$. In the case of Cournot competition this equals

$$
\begin{aligned}
\bar{p}^{C}(\underline{c}) & =\frac{1}{2} p\left(\left(1+\frac{1}{3} \sqrt{5}\right) \frac{a-\bar{c}}{2 b}\right)+\frac{1}{2} p\left(\frac{a-\underline{c}}{2 b}\right) \\
& =\frac{1}{4}(a+\bar{c})-\frac{1}{12} \sqrt{5}(a-\bar{c})+\frac{1}{4}(a+\underline{c}) .
\end{aligned}
$$

The average price for a low cost incumbent under Stackelberg competition equals

$$
\begin{aligned}
\bar{p}^{S}(\underline{c}) & =\frac{1}{2} p\left(\left(1+\frac{1}{2} \sqrt{2}\right) \frac{a-\bar{c}}{2 b}\right)+\frac{1}{2} p\left(\frac{a-\underline{c}}{2 b}\right) \\
& =\frac{1}{4}(a+\bar{c})-\frac{1}{8} \sqrt{2}(a-\bar{c})+\frac{1}{4}(a+\underline{c}) .
\end{aligned}
$$

With a high cost incumbent, the price in the first period equals the price related with its monopoly quantity: $p\left(q^{m}(\bar{c})\right)=(a+\bar{c}) / 2$. In the post-entry period the market price will be either the Cournot or the Stackelberg price, given that both firms have high costs: we define these prices by $p^{C}(\bar{c}, \bar{c})$ and $p^{S}(\bar{c}, \bar{c})$. We thus have

$$
\begin{aligned}
& \bar{p}^{C}(\bar{c})=\frac{a+\bar{c}}{4}+\frac{1}{2} p^{C}(\bar{c}, \bar{c})=\frac{5 a+7 \bar{c}}{12} \\
& \bar{p}^{S}(\bar{c})=\frac{a+\bar{c}}{4}+\frac{1}{2} p^{S}(\bar{c}, \bar{c})=\frac{3 a+5 \bar{c}}{8} .
\end{aligned}
$$


Note that for the expected average price in the Cournot model, which we will call $E\left(\bar{p}^{C}\right)$ and for the expected average price in the Stackelberg model, $E\left(\bar{p}^{S}\right)$, we have

$$
\begin{aligned}
& E\left(\bar{p}^{C}\right)=\rho \cdot \bar{p}^{C}(\underline{c})+(1-\rho) \cdot \bar{p}^{C}(\bar{c}) \\
& E\left(\bar{p}^{S}\right)=\rho \cdot \bar{p}^{S}(\underline{c})+(1-\rho) \cdot \bar{p}^{S}(\bar{c})
\end{aligned}
$$

When we equate $E\left(\bar{p}^{C}\right)$ and $E\left(\bar{p}^{S}\right)$ we find that the two are equal if

$$
\tilde{\rho}=\frac{1}{1+2 \sqrt{5}-3 \sqrt{2}} .
$$

Since the average Stackelberg price is higher for a low cost incumbent and lower for a high cost incumbent, we thus have that the expected average price is higher when $\rho>\tilde{\rho}$

4. Suppose conditions 1 and 2 do not hold and we have $\rho^{* S}<\rho<\rho^{* C}$. We then have that in the Stackelberg model we are in the upper row of table 2, whereas in the Cournot model we are in the lower row. We thus have that a low cost incumbent in the Stackelberg model sets its monopoly quantity in both periods, whereas in the Cournot model it sets its limit price in the pre-entry period. The average price for a low cost incumbent is thus higher in the Stackelberg model.

A high cost incumbent in the Stackelberg model sets $q^{m}(\underline{c})$ in the pre-entry period, and deters entry. The average price then equals

$$
\bar{p}^{S}(\bar{c})=\frac{1}{2}\left\{\frac{a+\underline{c}}{2}+\frac{a+\bar{c}}{2}\right\} .
$$

A high cost incumbent in the Cournot model sets $q^{m}(\bar{c})$ in the pre-entry period and induces entry, which then yields a Cournot price. The average price then equals

$$
\bar{p}^{C}(\bar{c})=\frac{1}{2}\left\{\frac{a+\bar{c}}{2}+\frac{a+2 \bar{c}}{3}\right\} .
$$

It is now easy to show that $\Delta c<(a-\bar{c}) / 3$ is sufficient for $\bar{p}^{S}(\bar{c})>\bar{p}^{C}(\bar{c})$. Since we already showed that in this case $\bar{p}^{S}(\underline{c})>\bar{p}^{C}(\underline{c})$, we have proven that condition 3 in the theorem is sufficient for the theorem to hold.

5. Now suppose $\rho^{* S}<\rho<\rho^{* C}$, but $\Delta c>(a-\bar{c}) / 3$. For a low cost incumbent we have

$$
\bar{p}^{S}(\underline{c})=\frac{a+\underline{c}}{2},
$$

whereas $\bar{p}^{C}(\underline{c})$ is the same as the one in $(28)$;

$$
\bar{p}^{C}(\underline{c})=\frac{1}{4}(a+\bar{c})-\frac{1}{12} \sqrt{5}(a-\bar{c})+\frac{1}{4}(a+\underline{c}) .
$$

Using (30), and (33) through (36), we can show that $E\left(\bar{p}^{C}\right)=E\left(\bar{p}^{S}\right)$ if

$$
\bar{\rho}=\frac{3 \Delta c-(a-\bar{c})}{(\sqrt{5}-1)(a-\bar{c})} .
$$

We thus have that expected average price under Stackelberg competition is higher if $\rho>\bar{\rho}$,

which proves the theorem. 